\title{
Three-dimensional thermal weight function method for the interface crack problems in bimaterial structures under a transient thermal loading
}

\author{
Huaping Wu, Long Li, Guozhong Chai, Fan Song \& Takayuki Kitamura
}

To cite this article: Huaping Wu, Long Li, Guozhong Chai, Fan Song \& Takayuki Kitamura (2016) Three-dimensional thermal weight function method for the interface crack problems in bimaterial structures under a transient thermal loading, Journal of Thermal Stresses, 39:4, 371-385

To link to this article: http://dx.doi.org/10.1080/01495739.2016.1152108

曲 Published online: 22 Mar 2016.

Submit your article to this journal ๘

View related articles $\widetilde{ }$

View Crossmark data $־$ 


\title{
Three-dimensional thermal weight function method for the interface crack problems in bimaterial structures under a transient thermal loading
}

\author{
Huaping $\mathrm{Wu}^{\mathrm{a}, \mathrm{b}}$, Long $\mathrm{Li}^{\mathrm{c}}$, Guozhong Chai ${ }^{\mathrm{a}}$, Fan Song ${ }^{\mathrm{c}}$, and Takayuki Kitamura ${ }^{\mathrm{b}}$ \\ ${ }^{a}$ Key Laboratory of E\&M, Ministry of Education \& Zhejiang Province, Zhejiang University of Technology, Hangzhou, China; \\ ${ }^{b}$ Department of Mechanical Engineering and Science, Kyoto University, Nishikyo-ku, Kyoto, Japan; 'State Key Laboratory \\ of Nonlinear Mechanics, Institute of Mechanics, Chinese Academy of Sciences, Beijing, China
}

\begin{abstract}
Different from previous two-dimensional thermal weight function (TWF) method, a three-dimensional (3D) TWF method is proposed for solving elliptical interface crack problems in bimaterial structures under a transient thermal loading. The present 3D TWF method based on the Betti's reciprocal theorem is a powerful tool for dealing with the transient thermal loading due to the stress intensity factors (SIFs) of whole transient process obtained through the static finite element computation. Several representative examples demonstrate that the 3D TWF method can be used to predict the SIFs of elliptical interface crack subjected to transient thermal loading with high accuracy. Moreover, numerical results indicate that the computing efficiency can be enhanced when dealing with transient problems, especially for large amount of time instants.
\end{abstract}

\section{ARTICLE HISTORY}

Received 28 April 2015

Accepted 18 May 2015

\section{KEYWORDS}

Bimaterial structures; 3D thermal weight function method; interface crack; stress intensity factor (SIF); transient thermal loading

\section{Introduction}

In recent years, composite materials that can cope with extreme thermal environmental conditions have been given considerable attention in structural applications. Lots of composite materials and structures used in combustion chambers, turbines, nuclear reactors, multilayer ceramic structures, and electronic packaging structures are subjected to transient thermal loadings during their service life. Unfortunately, the cracks at the interface between the dissimilar materials affect the strength of the composite structure significantly due to the high thermal stresses near the interface crack tips which arises from the thermal mismatch. From the structural integrity of composite materials point of view, the accurate assessment of interface fracture behavior is very important.

In the classical study of thermoelastic crack problems, a lot of studies have been conducted, both analytically and numerically. The analytical solutions are available only for very limited cases [1-14], wherein the cracked bodies are assumed to be infinite and subjected to special thermal loading conditions. It is difficult to derive the exact solutions for cracked bodies of finite dimension. On numerical aspect, a lot of studies have been performed based on the finite element method [15-25] and the boundary element method [26-29] to solve the interface crack problems of finite dimension. The methods mentioned above may be categorized as the direct methods, which have a common disadvantage. Repeated finite element analysis or boundary element analysis will be needed for the same cracked body subjected to different thermal loadings, especially when dealing with transient thermal problems wherein a large amount of computation works will be carried out for a great number of time instants and for different thermal shock conditions [30, 31]. 
The thermal weight function (TWF) method proposed by Tsai and Ma [32] provides an alternative efficient method to deal with a homogeneous cracked body under transient thermal loadings. The TWF method is extended from the weight function concept for mechanical loading. The weight function concept was introduced by Bueckner [33] and Rice [34]. Subsequently, many contributors extended the theory of weight function to a more generalized form. To distinguish from the TWF, the weight function for mechanical loading could be named as mechanical weight function, which provides an efficient approach for calculating the stress intensity factors (SIFs) for a cracked body subjected to mechanical loadings. The unique advantage of this method is that the weight function is a universal function for a given crack configuration and body geometry. Once the weight function is obtained from a specific crack tip in a given body, the SIFs for that crack tip can be determined directly for any other complicated mechanical loading by a simple integration [35]. However, the repeated stress analysis, to obtain the tractions along the crack line for an uncracked body, is inevitable for dealing with the transient thermal problems [36]. Distinguished from the direct methods and the mechanical weight function method, the TWF method can be applied to solve the transient problems efficiently. The TWF is also a universal function which is dependent only on the crack configuration and body geometry and independent of thermal loading. Since the TWF is independent of time during thermal shock, the whole variation of the transient SIFs can be directly determined through integration of the products of the TWF and the transient temperature fields. The amount of calculation can be greatly reduced because the repeated determination of the distributions of stress or displacement fields for individual time instants is avoided.

In view of these advantages and considering that most cracks in engineering practice are threedimensional (3D) cracks, this paper aims to propose a 3D TWF method to deal with thermal problems in the elliptical interface crack system of bimaterial structures. The basic equation of the present 3D TWF method for 3D interface crack problems is derived based on the Betti's reciprocal theorem. The multiple virtual crack extension technique is applied to calculate the SIF distributions along 3D interface crack fronts subjected to thermal loading. The present method is validated through analyzing several representative examples based on the assumption that the thermal-elastic problem is decoupled and quasi-static, the thermoelastic constants are independent on the temperature, and the crack does not affect the temperature distribution. In addition, the effect of the crack depth on the SIFs has been analyzed, and some useful results for engineering application have been obtained.

\section{Formulation}

The 3D TWF for an interface crack of bimaterial can be determined by a known solution of fundamental reference loading system. Consider the geometrically equivalent configurations shown in Figures 1a and $1 \mathrm{~b}$. These configurations are subjected to prescribed traction $\boldsymbol{t}^{*}$ on boundary $\sum_{\mathrm{t}}$, prescribed displacement $\boldsymbol{u}^{*}$ on boundary $\sum_{\mathrm{u}}$, and body force $\boldsymbol{f}^{*}$ and thermal load $\Theta^{*}$ are designated as case $(r)$ and $(p)$, respectively. Here, the thermal load $\Theta^{*}=T_{0}-T, T_{0}$ is a reference temperature and $T$ is the current temperature. The case $(r)$ is the fundamental reference loading system wherein the SIFs variations of Mode I, Mode II, and Mode III along the interface crack front are known. The case $(p)$ is an unknown loading system wherein the SIFs along the crack front are to be solved.

Using Betti's reciprocal theorem for the geometry with interface crack face $S$, one obtains

$$
\begin{aligned}
& \int_{\Sigma_{\mathrm{t}}} \boldsymbol{t}^{*(r)} \cdot \boldsymbol{u}^{(p)}(S) d \Sigma-\int_{\Sigma_{\mathrm{u}}} \boldsymbol{u}^{*(r)} \cdot \boldsymbol{t}^{(p)}(S) d \Sigma \\
& \quad+\int_{V} \boldsymbol{f}^{*(r)} \cdot \boldsymbol{u}^{(p)}(S) d V+\sum_{j=1}^{2} \int_{V_{j}} \alpha_{j} \Theta^{*(r)} \cdot \sigma_{k k}^{(p)}(S) d V \\
& =\int_{\Sigma_{\mathrm{t}}} \boldsymbol{t}^{*(p)}(S) \cdot \boldsymbol{u}^{(r)}(S) d \Sigma-\int_{\Sigma_{\mathrm{u}}} \boldsymbol{u}^{*(p)}(S) \cdot \boldsymbol{t}^{(r)}(S) d \Sigma \\
& \quad+\int_{V} \boldsymbol{f}^{*(p)}(S) \cdot \boldsymbol{u}^{(r)}(S) d V+\sum_{j=1}^{2} \int_{V_{j}} \alpha_{j} \Theta^{*(p)}(S) \cdot \sigma_{k k}^{(r)}(S) d V
\end{aligned}
$$



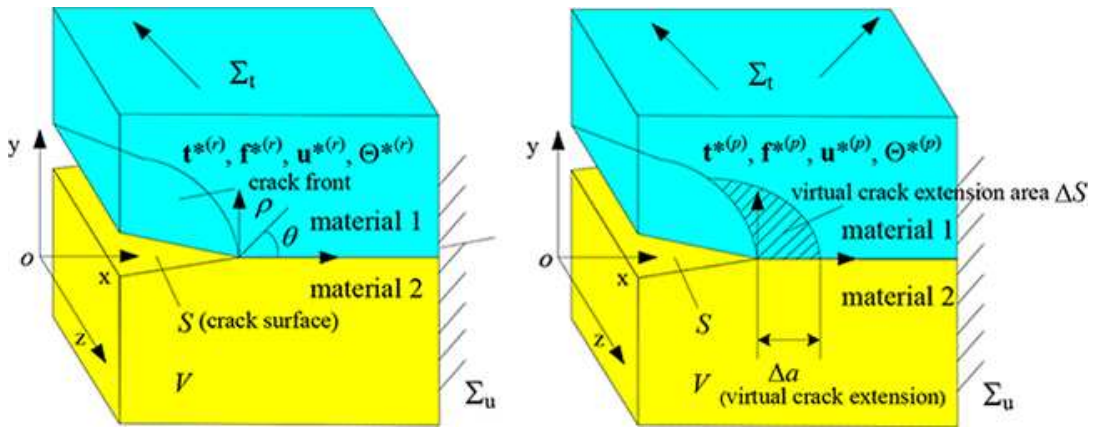

Figure 1. Configurations: (a) fundamental reference loading system ( $r$ ) and (b) loading system ( $p$ ) to be solved.

where $\alpha_{j}$ is the thermal expansion coefficient, $V_{j}$ is the volume of the cracked body surrounded by the surface $\sum$, and the subscripts $j=1$ or 2 refer to the material above or below the crack plane, $\Sigma=\sum_{\mathrm{t}} \cup \sum_{\mathrm{u}}$. The repeated indices imply the summing over their range.

If the interface crack face has a virtual crack extension $\Delta S$, the original traction on $\Delta S$ in system $(r)$ can be treated as a part of traction $\boldsymbol{t}^{*(r)}$ for the new system. The opposite traction is applied on the crack face $\Delta S$ to keep the crack opening displacements to be 0 . Applying Betti's reciprocal theorem for the geometry with the current crack face $S+\Delta S$, we can get an equation in Taylor expansion as

$$
\begin{aligned}
\int_{\Sigma_{\mathrm{t}}} & \boldsymbol{t}^{*(r)} \cdot\left[\boldsymbol{u}^{(p)}(S)+\frac{\partial \boldsymbol{u}^{(p)}}{\partial S} \Delta S\right] d \Sigma+\int_{\Sigma_{\Delta S}} \boldsymbol{t}^{*(r)} \cdot\left[\boldsymbol{u}^{(p)}(S)+\frac{\partial \boldsymbol{u}^{(p)}}{\partial S} \Delta S\right] d \Sigma \\
& -\int_{\Sigma_{\mathrm{u}}} \boldsymbol{u}^{*(r)} \cdot\left[\boldsymbol{t}^{(p)}(S)+\frac{\partial \boldsymbol{t}^{(p)}}{\partial S} \Delta S\right] d \Sigma \\
& +\int_{V} \boldsymbol{f}^{*(r)} \cdot\left[\boldsymbol{u}^{(p)}(S)+\frac{\partial \boldsymbol{u}^{(p)}}{\partial S} \Delta S\right] d V+\sum_{j=1}^{2} \int_{V_{j}} \alpha_{j} \Theta^{*(r)} \cdot\left[\sigma_{\mathrm{kk}}^{(p)}(S)+\frac{\partial \sigma_{\mathrm{kk}}^{(p)}}{\partial S} \Delta S\right] d V \\
= & \int_{\Sigma_{\mathrm{t}}}\left[\boldsymbol{t}^{*(p)}(S)+\frac{\partial \mathrm{t}^{*(p)}}{\partial S} \Delta S\right] \cdot \mathrm{u}^{(r)}(S) d \Sigma-\int_{\Sigma_{\mathrm{u}}}\left[\mathrm{u}^{*(p)}(S)+\frac{\partial \mathrm{u}^{*(p)}}{\partial S} \Delta S\right] \cdot \mathrm{t}^{(r)}(S) d \Sigma \\
& +\int_{V}\left[\boldsymbol{f}^{*(p)}(S)+\frac{\partial \mathrm{f}^{*(p)}}{\partial S} \Delta S\right] \cdot \mathrm{u}^{(r)}(S) d V \\
& +\sum_{j=1}^{2} \int_{V_{j}}\left[\alpha_{j} \Theta^{*(p)}(S)+\frac{\partial\left(\alpha_{j} \Theta^{*(p)}\right)}{\partial S} \Delta S\right] \cdot \sigma_{\mathrm{kk}}^{(r)}(S) d V
\end{aligned}
$$

Differentiating Eq. (1) with respect to $S$ yields

$$
\begin{aligned}
& \frac{1}{\Delta S}\left[-\int_{\Sigma_{\Delta S}} \boldsymbol{t}^{*(r)} \cdot \boldsymbol{u}^{(p)} d \Sigma\right]=\int_{\Sigma_{\mathrm{t}}} \boldsymbol{t}^{*(p)} \cdot \frac{\partial \boldsymbol{u}^{(r)}}{\partial S} d \Sigma-\int_{\Sigma_{\mathrm{u}}} \boldsymbol{u}^{*(p)} \cdot \frac{\partial \boldsymbol{t}^{(r)}}{\partial S} d \Sigma \\
& +\int_{V} \boldsymbol{f}^{*(p)} \cdot \frac{\partial \boldsymbol{u}^{(r)}}{\partial S} d V+\sum_{j=1}^{2} \int_{V_{j}} \alpha_{j} \Theta^{*(p)} \cdot \frac{\partial \sigma_{\mathrm{kk}}^{(r)}}{\partial S} d V
\end{aligned}
$$

By considering thermal loading, Eq. (3) becomes

$$
\frac{1}{\Delta S}\left[-\int_{\Sigma_{\Delta S}} \boldsymbol{t}^{*(r)} \cdot \boldsymbol{u}^{(p)} d \Sigma\right]=\sum_{j=1}^{2} \int_{V_{j}} \alpha_{j} \Theta^{*(p)} \cdot \frac{\partial \sigma_{\mathrm{kk}}^{(r)}}{\partial S} d V
$$


The stresses, $\sigma_{y y}, \sigma_{x y}$, and $\sigma_{y z}$, in the vicinity of the crack front can be expressed in local crack tip coordinate system as [37]:

$$
\begin{aligned}
\left(\sigma_{\mathrm{yy}}+\mathrm{i} \sigma_{\mathrm{xy}}\right)_{\theta=0} & =\frac{K_{1}+i K_{2}}{\sqrt{2 \pi \rho}}\left(\frac{\rho}{l_{0}}\right)^{i \varepsilon} \\
\sigma_{\mathrm{yz}}\left(\rho, 0^{+}\right) & =\left(0, \frac{-K_{\mathrm{III}}}{\sqrt{2 \pi \rho}}\right) \\
\sigma_{\mathrm{yz}}\left(\rho, 0^{-}\right) & =\left(0, \frac{K_{\mathrm{III}}}{\sqrt{2 \pi \rho}}\right)
\end{aligned}
$$

where $i=\sqrt{-1}, \rho$ and $\theta$ are the polar coordinates, and the values $0^{+}$and $0^{-}$of $\theta$ denote the upper and lower crack faces, respectively. The bimaterial constant $\epsilon$ is defined as

$$
\varepsilon=\frac{1}{2 \pi} \ln \left(\frac{\kappa_{1} \mu_{2}+\mu_{1}}{\kappa_{2} \mu_{1}+\mu_{2}}\right)
$$

where $\kappa_{\mathrm{j}}=3-4 v_{\mathrm{j}}$ for plane strain and $\left(3-v_{\mathrm{j}}\right) /\left(1+v_{\mathrm{j}}\right)$ for generalized plane stress, and $\mu_{\mathrm{j}}$ and $v_{j}$ are the shear modulus and Poisson's ratio.

The displacements, $u_{x}, u_{y}$, and $u_{z}$, in the vicinity of the crack front are [37]:

$$
\begin{aligned}
\delta u_{\mathrm{y}}+i \delta u_{\mathrm{x}} & =\frac{K_{1}+i K_{2}}{2(1+2 i \varepsilon) \cos h(\varepsilon \pi)}\left(\frac{\kappa_{1}+1}{\mu_{1}}+\frac{\kappa_{2}+1}{\mu_{2}}\right) \sqrt{\frac{\rho}{2 \pi}}\left(\frac{\rho}{l_{0}}\right)^{i \varepsilon} \\
u_{\mathrm{z}}\left(\rho, 0^{+}\right) & =\left(0, \frac{K_{\mathrm{III}}}{\mu_{1}} \sqrt{\frac{2 \rho}{\pi}}\right) \\
u_{\mathrm{z}}\left(\rho, 0^{-}\right) & =\left(0, \frac{-K_{\mathrm{III}}}{\mu_{2}} \sqrt{\frac{2 \rho}{\pi}}\right)
\end{aligned}
$$

By substituting Eqs. (5) and (7) into Eq. (4), the left side of Eq. (4) reduces to

$$
\begin{aligned}
& \frac{1}{\Delta S}\left[-\int_{\Sigma_{\Delta S}} \boldsymbol{t}^{*(r)} \cdot \boldsymbol{u}^{(p)} d \Sigma\right]=\frac{1}{\Delta S}\left\{\begin{array}{l}
\Re\left[\int_{\Sigma_{\Delta S}}\left(\sigma_{\mathrm{yy}}+i \sigma_{\mathrm{xy}}\right)^{*(r)}(\rho) \cdot\left(u_{\mathrm{y}}-i u_{\mathrm{x}}\right)^{(p)}(\Delta a-\rho) d \Sigma\right] \\
-\int_{\Sigma_{\Delta S}}\left(\sigma_{\mathrm{yz}}\right)^{*(r)}(\rho) \cdot u_{\mathrm{z}}^{(p)}(\Delta a-\rho) d \Sigma
\end{array}\right\} \\
& \quad=\frac{1}{\Delta S}\left\{\begin{array}{l}
\Re\left[\int_{\Delta S} \frac{4\left[\left(K_{1}^{(r)} K_{1}^{(p)}+K_{2}^{(r)} K_{2}^{(p)}\right)+i\left(K_{2}^{(r)} K_{1}^{(p)}-K_{1}^{(r)} K_{2}^{(p)}\right)\right]}{H^{*} \pi \cos h(\varepsilon \pi)(1-2 i \varepsilon)}\left(\frac{\Delta a-\rho}{\rho}\right)^{1 / 2-i \varepsilon} d S\right] \\
+\int_{\Delta S} \frac{4 K_{\mathrm{III}}^{(r)} K_{\mathrm{III}}^{(p)}}{\mu^{*} \pi} \sqrt{\frac{(\Delta a-\rho)}{\rho}} d S
\end{array}\right\}
\end{aligned}
$$

Equation (8) is the integral only along one side of the crack face, where $d S$ is the increment of the integration over $\Delta S$, and $\mu^{*}$ and $H^{*}$ are effective shear modulus and Young's modulus given by

$$
\begin{aligned}
\frac{1}{\mu^{*}} & =\frac{1}{2}\left(\frac{1}{\mu_{1}}+\frac{1}{\mu_{2}}\right) \\
\frac{1}{H^{*}} & =\frac{1}{2}\left(\frac{1}{H_{1}}+\frac{1}{H_{2}}\right)
\end{aligned}
$$


Since $\sigma_{k k}$ is sensitive to the crack size, the partial derivative in the equation is difficult to be evaluated. For 3D elastic problems,

$$
\sigma_{\mathrm{kk}}=\frac{E}{1-2 v} \varepsilon_{\mathrm{kk}}=\frac{E}{1-2 v}\left(\frac{\partial u}{\partial x}+\frac{\partial v}{\partial y}+\frac{\partial w}{\partial z}\right)
$$

By applying Eq. (10), the Green's theorem and the Gauss's theorem (Eq. (4)) can be expressed as

$$
\begin{aligned}
\frac{1}{\Delta S} & {\left[-\int_{\Sigma_{\Delta S}} \boldsymbol{t}^{*(r)} \cdot \boldsymbol{u}^{(p)} d \Sigma\right] } \\
= & \sum_{j=1}^{2} \frac{E_{j}}{1-2 v_{j}}\left[\oint \int_{\sum_{j}} \alpha_{j} \Theta^{*(p)}\left(\frac{\partial u^{(r)}}{\partial S} \cos \alpha+\frac{\partial v^{(r)}}{\partial S} \cos \beta+\frac{\partial w^{(r)}}{\partial S} \cos \gamma\right) d \sum\right. \\
& \left.-\iiint_{V_{j}}\left(\frac{\partial\left(\alpha_{j} \Theta^{*(p)}\right)}{\partial x} \frac{\partial u^{(r)}}{\partial S}+\frac{\partial\left(\alpha_{j} \Theta^{*(p)}\right)}{\partial y} \frac{\partial v^{(r)}}{\partial S}+\frac{\partial\left(\alpha_{j} \Theta^{*(p)}\right)}{\partial z} \frac{\partial w^{(r)}}{\partial S}\right) d V\right]
\end{aligned}
$$

where $\cos \alpha, \cos \beta$, and $\cos \gamma$ are direction cosines of the normal vector of the boundary surface. Substituting Eq. (8) into Eq. (11) yields

$$
\begin{aligned}
\frac{1}{\Delta S}\left\{\begin{array}{l}
\Re\left[\int_{\Delta S} \frac{4\left[\left(K_{1}^{(r)} K_{1}^{(p)}+K_{2}^{(r)} K_{2}^{(p)}\right)+i\left(K_{2}^{(r)} K_{1}^{(p)}-K_{1}^{(r)} K_{2}^{(p)}\right)\right]}{H^{*} \pi \cos h(\varepsilon \pi)(1-2 i \varepsilon)}\left(\frac{\Delta a-\rho}{\rho}\right)^{1 / 2-i \varepsilon} d S\right] \\
+\int_{\Delta S} \frac{4 K_{\mathrm{III}}^{(r)} K_{\mathrm{III}}^{(p)}}{\mu^{*} \pi} \sqrt{\frac{(\Delta a-\rho)}{\rho}} d S
\end{array}\right\} \\
=\sum_{j=1}^{2} \frac{E_{j}}{1-2 v_{j}}\left[\oint \int_{\sum_{j}} \alpha_{j} \Theta^{*(p)}\left(\frac{\partial u^{(r)}}{\partial S} \cos \alpha+\frac{\partial v^{(r)}}{\partial S} \cos \beta+\frac{\partial w^{(r)}}{\partial S} \cos \gamma\right) d \sum\right. \\
\left.\quad-\iiint_{V_{j}}\left(\frac{\partial\left(\alpha_{j} \Theta^{*(p)}\right)}{\partial x} \frac{\partial u^{(r)}}{\partial S}+\frac{\partial\left(\alpha_{j} \Theta^{*(p)}\right)}{\partial y} \frac{\partial v^{(r)}}{\partial S}+\frac{\partial\left(\alpha_{j} \Theta^{*(p)}\right)}{\partial z} \frac{\partial w^{(r)}}{\partial S}\right) d V\right]
\end{aligned}
$$

Equation (12) is the basic equation of TWF method for a 3D crack of bimaterial interface. As the SIFs of different crack opening modes for a 3D crack cannot be simply separated from the integral since they vary along the interface crack front, the following research will be focused on how to separate the SIFs of different modes.

\section{Finite element implementation of the 3D TWF method}

To separate SIFs from the integral in Eq. (12), the multiple virtual crack extension technique will be applied. For elliptical cracks, an effective crack length $a_{\phi}$ is adopted along the interface crack front (cf. Figure 2),

$$
a_{\varphi}=\sqrt{\sin ^{2} \varphi+(a / c)^{2} \cos ^{2} \varphi} \cdot a
$$

where $a$ and $c$ represent the length of semiminor axis and semimajor axis, respectively, and $\phi$ is the effective angle of the elliptical crack. A basic virtual crack extension mode $\Delta a_{\varphi}^{0}$ is defined based on the Eq. (13) as

$$
\Delta a_{\varphi}^{0}=\sqrt{\sin ^{2} \varphi+(a / c)^{2} \cos ^{2} \varphi} \cdot \Delta a
$$

Several linearly independent virtual crack extension modes $\Delta a_{\phi}^{j}$ are introduced, i.e.,

$$
\Delta a_{\varphi}^{j}=\omega_{j}(\varphi) \Delta a_{\varphi}^{0} \quad(j=1,2, \ldots, N)
$$




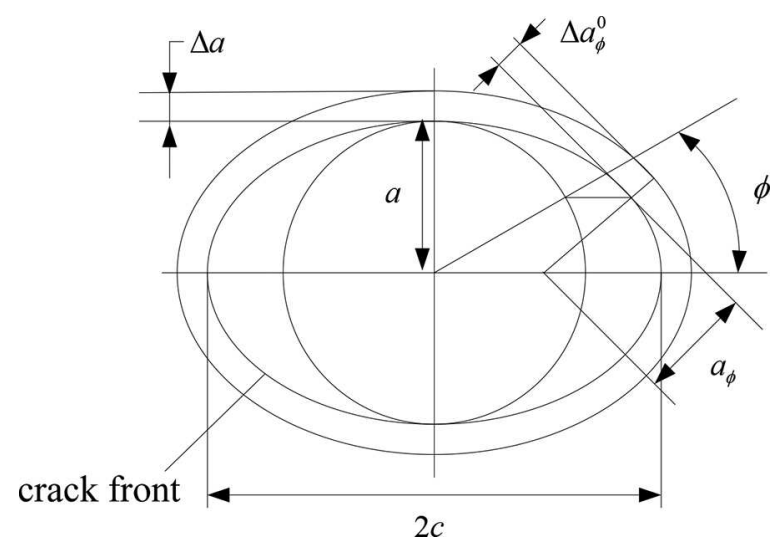

Figure 2. Schematic diagram of elliptical crack.

where $N$ is the number of the points on the interface crack front, $\omega_{j}(\phi)$ is the extension function satisfying the basic nature of shape function,

$$
\omega_{j}\left(\varphi_{i}\right)=\delta_{i j}=\left\{\begin{array}{ll}
1 & (i=j) \\
0 & (i \neq j)
\end{array} \quad \sum_{j=1}^{N} \omega_{j}(\varphi)=1\right.
$$

Thus, the SIFs can be directly written as

$$
\begin{aligned}
& K_{1}^{(p)}=\sum_{i=1}^{n} A_{\mathrm{I} i} \omega_{i}(\varphi) K_{1}^{(0)} \\
& K_{2}^{(p)}=\sum_{i=1}^{n} A_{\mathrm{II} i} \omega_{i}(\varphi) K_{2}^{(0)} \\
& K_{\mathrm{III}}^{(p)}=\sum_{i=1}^{n} A_{\mathrm{III} i} \omega_{i}(\varphi) K_{\mathrm{III}}^{(0)}
\end{aligned}
$$

where $A_{\mathrm{I} i}, A_{\mathrm{II} i}$, and $A_{\mathrm{III} i}$ are the coefficients to be determined, and $K_{1}^{(0)}, K_{2}^{(0)}$, and $K_{\mathrm{III}}^{(0)}$ are arbitrary distribution functions.

It should be noted that

$$
\begin{aligned}
& d S=d \rho d l \quad d l=c \sqrt{\sin ^{2} \varphi+(a / c)^{2} \cos ^{2} \varphi} d \varphi \quad \int_{0}^{\Delta a_{\varphi}} \sqrt{\frac{\left(\Delta a_{\varphi}-\rho\right)}{\rho}} d \rho=\frac{\pi}{2} \Delta a_{\varphi} \\
& \Re\left[\frac{1}{\Delta a_{\varphi}} \int_{0}^{\Delta a_{\varphi}} \frac{4\left[\left(K_{1}^{(r)} K_{1}^{(p)}+K_{2}^{(r)} K_{2}^{(p)}\right)+i\left(K_{2}^{(r)} K_{1}^{(p)}-K_{1}^{(r)} K_{2}^{(p)}\right)\right]}{H^{*} \pi \cos h(\varepsilon \pi)(1-2 i \varepsilon)}\left(\frac{\Delta a_{\varphi}-\rho}{\rho}\right)^{1 / 2-i \varepsilon} d \rho\right] \\
&=\frac{2\left(K_{1}^{(r)} K_{1}^{(p)}+K_{2}^{(r)} K_{2}^{(p)}\right)}{H^{*} \cos h^{2}(\pi \varepsilon)}
\end{aligned}
$$

where the last integral is recognized as the complex beta function $B(1 / 2+i \varepsilon, 3 / 2-i \varepsilon), l$ is the arc length along the interface crack front. And in view of Eqs. (14), (15), and (17), we can simplify the left 
side of Eq. (12) (denoted as $\left.I_{\text {left }}\right)$ as

$$
\left.\begin{array}{rl}
I_{\text {left }}= & \frac{\Delta a}{\Delta S_{j}} \int_{\varphi_{1}}^{\varphi_{N}} 2 c \omega_{i}(\varphi)\left[\begin{array}{l}
\frac{\left(\sum_{i=1}^{N} A_{\mathrm{Ii}} K_{1}^{(r)} K_{1}^{(0)}+\sum_{i=1}^{N} A_{\mathrm{II} i} K_{2}^{(r)} K_{2}^{(0)}\right)}{H^{*} \cosh ^{2}(\pi \varepsilon)} \\
+\frac{\sum_{i=1}^{N} A_{\mathrm{III} i} K_{\mathrm{III}}^{(r)} K_{\mathrm{III}}^{(0)}}{\mu^{*}}
\end{array}\right] \omega_{j}(\varphi) \\
& \times\left[\sin ^{2} \varphi+(a / c)^{2} \cos ^{2} \varphi\right] d \varphi
\end{array}\right]
$$

Here, $\varphi_{j}(j=1,2, \ldots, N)$ denotes the parametric angle of the elliptical crack.

The following expression of the partial derivatives is valid for a particular virtual crack extension $\Delta S_{j}$,

$$
\frac{\partial \boldsymbol{u}}{\partial S_{j}}=\frac{\partial \boldsymbol{u}}{\partial a} \frac{\Delta a}{\Delta S_{j}}
$$

Using the above expression of the partial derivatives in Eq. (12) and Eq. (20), we have

$$
\begin{aligned}
\int_{\varphi_{1}}^{\varphi_{N}} 2 c \omega_{i}(\varphi)\left[\frac{\left(\sum_{i=1}^{N} A_{\mathrm{II}} K_{1}^{(r)} K_{1}^{(0)}+\sum_{i=1}^{N} A_{\mathrm{III}} K_{2}^{(r)} K_{2}^{(0)}\right)}{\left.\frac{\sum_{i=1}^{N} A_{\mathrm{IIII}} K_{\mathrm{III}}^{(r)} \cosh _{\mathrm{III}}^{(0)}(\pi \varepsilon)}{\mu^{*}}+\right] \omega_{j}(\varphi)\left[\sin ^{2} \varphi+(a / c)^{2} \cos ^{2} \varphi\right] d \varphi}\right. \\
=\sum_{j=1}^{2} \frac{E_{j}}{1-2 v_{j}}\left[\oint \int_{\sum_{j}} \alpha_{j} \Theta^{*(p)}\left(\frac{\partial u^{(r)}}{\partial a} \cos \alpha+\frac{\partial v^{(r)}}{\partial a} \cos \beta+\frac{\partial w^{(r)}}{\partial a} \cos \gamma\right) d \sum\right. \\
\left.-\iiint_{V_{j}}\left(\frac{\partial\left(\alpha_{j} \Theta^{*(p)}\right)}{\partial x} \frac{\partial u^{(r)}}{\partial a}+\frac{\partial\left(\alpha_{j} \Theta^{*(p)}\right)}{\partial y} \frac{\partial v^{(r)}}{\partial a}+\frac{\partial\left(\alpha_{j} \Theta^{*(p)}\right)}{\partial z} \frac{\partial w^{(r)}}{\partial a}\right) d V\right]_{\Delta S_{j}} \\
=\sum_{j=1}^{2} \frac{E_{j}}{1-2 v_{j}}\left[\oint \int_{\sum_{j}} \alpha_{j} \Theta^{*(p)} \frac{\partial \boldsymbol{u}^{(r)}}{\partial a} \cdot n d \sum-\iiint_{V_{j}} \nabla\left(\alpha_{j} \Theta^{*(p)}\right) \cdot \frac{\partial \boldsymbol{u}^{(r)}}{\partial a} d V\right]_{\Delta S_{j}} \\
\quad(j=1,2, \ldots, N)
\end{aligned}
$$

where $\boldsymbol{u}^{(r)}=\left[\begin{array}{lll}u^{(r)} & v^{(r)} & w^{(r)}\end{array}\right]^{T}$ is the displacement vector of the fundamental reference loading system $(r), n=\left[\begin{array}{lll}\cos \alpha & \cos \beta & \cos \gamma\end{array}\right]^{T}$ is the normal vector of the surface $d \sum$. It can be found that, distinguished from the mechanical weight function method, if the temperature distribution is known, the whole variation of transient SIFs can be directly determined by the simple integration and the expensive time consumed for the numerical calculation of the thermal stress or displacement fields can be avoided at every transient time step.

Equation (21) is linear equations of the unknown coefficients. To obtain the solution of Eq. (21), three fundamental reference loading systems are required, that is, $r=1,2,3$. Then, Eq. (21) can be rewritten as

$$
\sum_{k=1}^{3} \sum_{i=1}^{3 N} C_{i j} A_{k i}=D_{j} \quad(j=1,2, \cdots, 3 N)
$$

Once the unknown coefficients, $A_{\mathrm{I} i}, A_{\mathrm{II} i}$, and $A_{\mathrm{III} i}$, are solved using Eq. (22), the distribution of the $K_{1}^{(p)}, K_{2}^{(p)}$, and $K_{\mathrm{III}}^{(p)}$ along the interface crack front can be obtained according to Eq. (17).

The partial derivatives of displacements and temperature with respect to coordinates can be obtained using the isoparametric elements and shape functions. The total derivatives of coordinates and displacements with respect to the characteristic crack length $a$ mentioned above can be obtained by the interpolation of nodal values. The stiffness derivative technique and virtual crack extension can be used to calculate the partial derivatives of the displacement with respect to $a$. 


\section{Verification and analyses}

In this section, several 3D bimaterial bodies with an elliptical interface crack under thermal load will be analyzed to demonstrate the practicability and accuracy of the 3D TWF method.

The thermoelastic problem is assumed to be decoupled and quasi-static, and the thermoelastic constants are independent of the temperature during the analysis procedure. Although the derivatives of displacement are strongly dependent on the reference loading system, the weight function is load independent for a given crack configuration with prespecified constrained conditions. It should be kept in mind that the fundamental reference loading systems must be linearly independent.

Preprocessing data for the 3D TWF method can be obtained using finite element computations. For the mechanical analysis, the 20-node isoparametric element (SOLID95 in ANSYS and in ABAQUS) is used over most of mesh and 1/4 node singular triangular prism-shaped element is used around the crack tip to get the reference SIFs accurately. Further, the 20-node C3D20T element in ABAQUS was used when dealing with the thermal analysis. The material properties used in these examples are shown in Table 1.

\section{Semi-infinite plate with an elliptical surface crack}

A semi-infinite plate with an elliptical surface crack subjected to a thermal load is analyzed initially (Figure 3). Three different boundary conditions are examined, i.e., the uniform heating, the cooling shock, and the instantaneous heat source. To simulate a semi-infinite plate, the following initial geometric conditions are adopted: $L=H=2 W=100 c$ and $a / c=0.5$. Due to the symmetry of the structure and boundary conditions, only the right half is modeled.

The finite element model contains 49428 20-node isoparametric elements with 211060 nodes. The finite element model is used for the displacement analysis of the fundamental reference loading systems to get the reference SIFs and to calculate TWFs. The model is also used for the thermal analysis to obtain the temperature distribution and the thermal SIFs which are adopted as the reference values to compare with the values of the SIFs obtained using the TWF method.

\section{Uniform heating}

The semi-infinite plate is subjected to uniform heating of $100^{\circ} \mathrm{C}$ on its front face, and the other free faces are insulated.

Figure 4 shows the SIFs calculated using the 3D TWF method and those obtained by the interaction integral method $[38,39] . K_{N}(I)$ and $K_{N}(T)$ denote the SIFs calculated by interaction integral method and TWF method, respectively. Here, $N=1,2$, and 3 . The SIFs will be normalized by $K_{0}$ which is defined as

$$
K_{0}=\frac{E_{1} \alpha_{1} \Theta^{*}}{1-v_{1}} \sqrt{\pi W}
$$

It can be seen that the results obtained by the 3D TWF method and interaction integral method are in close agreement. The differences between two methods at most locations on the crack front are less than or about $1.5 \%$.

Table 1. Material properties used in these examples.

\begin{tabular}{lcc}
\hline Materials parameter & Material 1 & Material 2 \\
\hline Young's modulus $(\mathrm{MPa})$ & $2.0 \mathrm{e} 5$ & $1.68 \mathrm{e} 5$ \\
Poisson's ratio & 0.23 & 0.31 \\
Thermal expansion coefficient $\left(1 /{ }^{\circ} \mathrm{C}\right)$ & $0.102 \mathrm{e}-4$ & $0.137 \mathrm{e}-4$ \\
Material density $\left(\mathrm{kg} / \mathrm{mm}^{3}\right)$ & $5.9 \mathrm{e}-6$ & $7.289 \mathrm{e}-6$ \\
Coefficient of $\mathrm{heat} \mathrm{conduction}\left(\mathrm{W} / \mathrm{mm}^{\circ} \mathrm{C}\right)$ & 2.2 & 48.9 \\
Specific heat $\left(\mathrm{J} /\left({ }^{\circ} \mathrm{C} \mathrm{kg}\right)\right)$ & 460.6 & 418.4 \\
\hline
\end{tabular}




\section{Cooling shock}

A transient fracture problem is considered in this part, the semi-infinite plate is under cooling shock $T=-100^{\circ} \mathrm{C}$ on its front face, and the other free faces are insulated.

To verify the accuracy of the results obtained from the 3D TWF method, the results for several time instants during the procedure of cooling shock are extracted for comparison (Figure 5). The SIFs are also normalized by $K_{0}$. It has been found that all the SIFs determined by the proposed TWF method agree well with those from interaction integral method, with the maximum relative errors of $3.6 \%$.

\section{Instantaneous heat source}

The third example is a semi-infinite plate in which an instantaneous heat source of strength $Q$ existent as shown in Figure 3. All free surfaces are insulated.

Figure 6 shows a comparison of the normalized SIFs obtained by the proposed method and interaction integral method for different time instants. The $\Theta^{*}$ in Eq. (23) is equal to the strength $Q$. The results

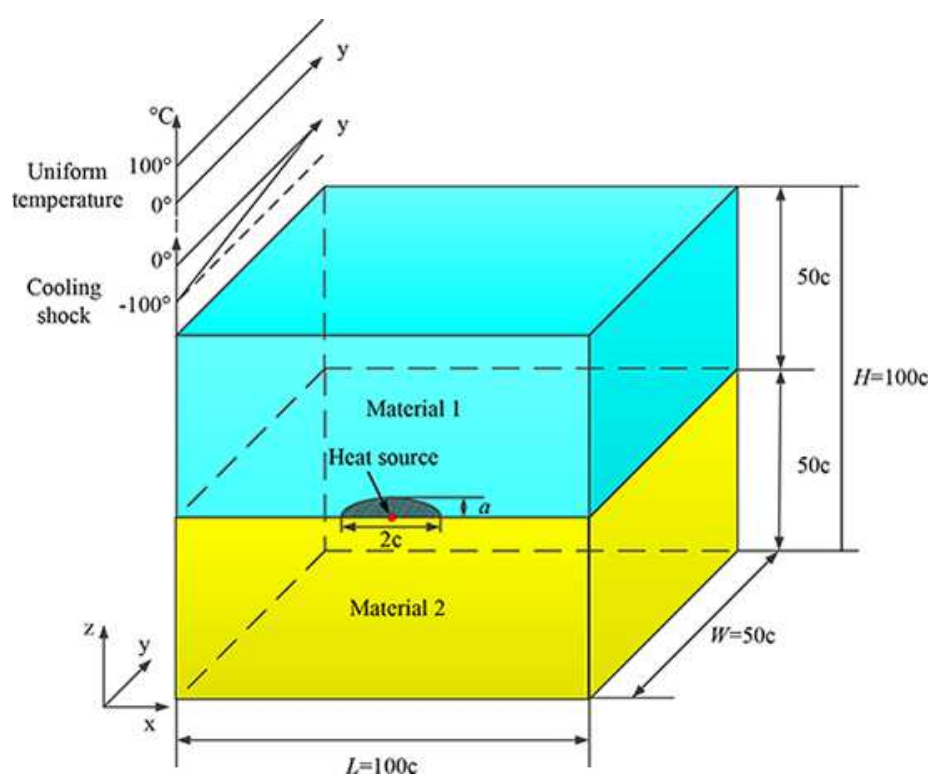

Figure 3. A semi-infinite plate with an elliptical surface crack under thermal load.

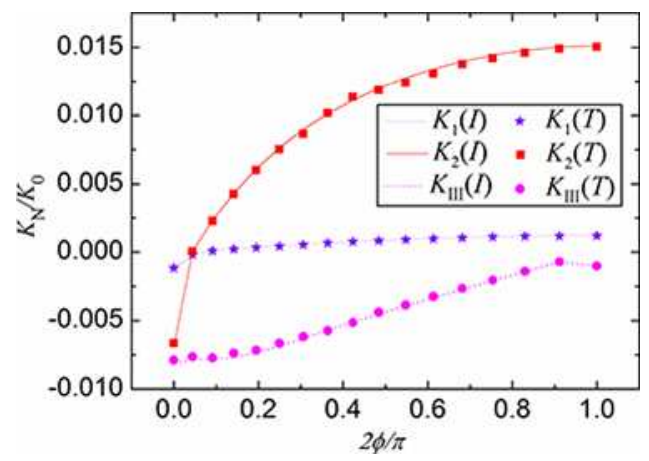

Figure 4. Comparison of stress intensity factors obtained from interaction integral method and TWF method for the case of uniform heating. TWF: thermal weight function. 


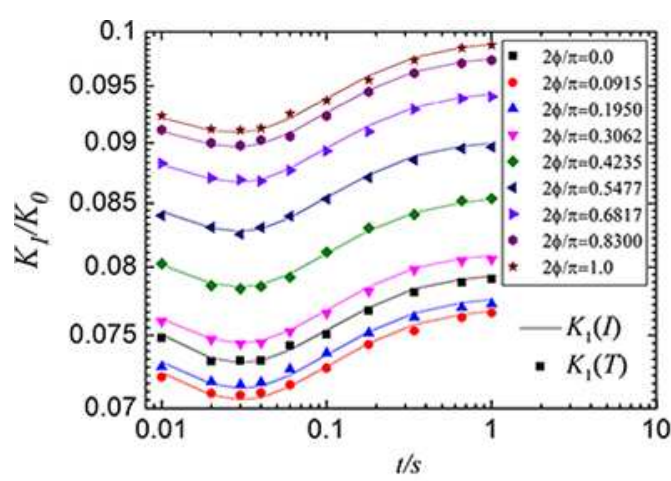

(a)

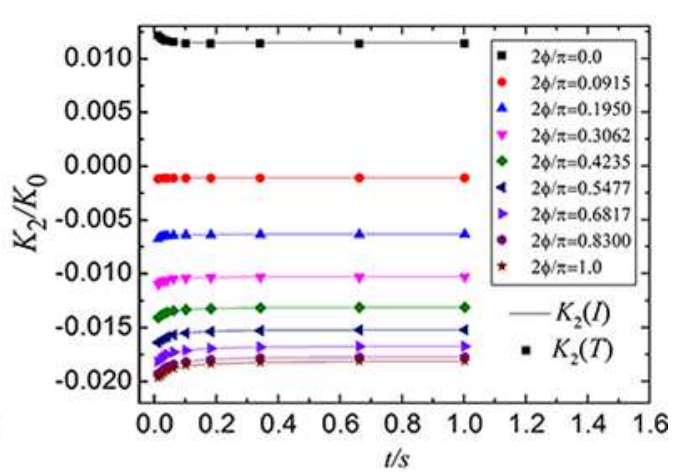

(b)

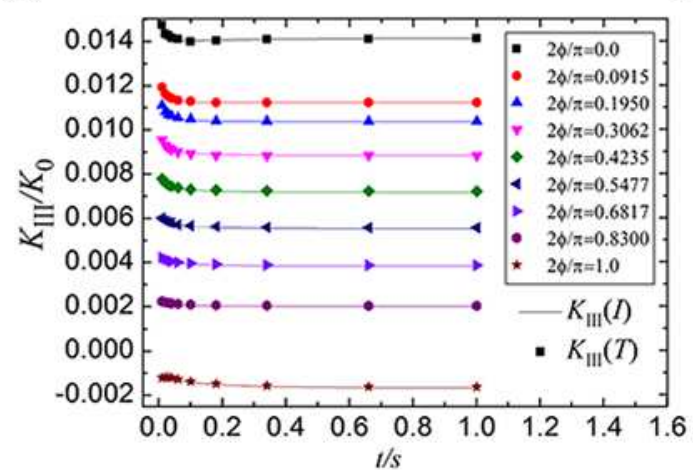

(c)

Figure 5. Comparison of stress intensity factors obtained from interaction integral method and TWF method for the case of cooling shock. TWF: thermal weight function.

obtained by these two methods show excellent agreement with each other. The relative errors between the SIFs obtained by these two methods are generally not excess $2.0 \%$ with the maximum of $3.7 \%$.

\section{Finite thermal barrier coating plate with a quarter-elliptical interface crack under cooling shock}

We next analyze a finite thermal barrier coating plate with a quarter-elliptical interface crack as shown in Figure 7. The bimaterial plate with a quarter-elliptical interface crack is fixed at the bottom end and subjected to cooling shock $T=-100^{\circ} \mathrm{C}$ on its top face. The other faces are insulated. The initial geometric boundary conditions are

$$
a / W=0.3, \quad a / c=0.5, \quad L / c=5, \quad H_{C}=L, \quad H_{C} / H_{S}=0.1
$$

The finite element model contains 397620 -node isoparametric elements and 17942 nodes.

The comparison of the results computed by these two methods is shown in Figure 8 . The results obtained by the 3D TWF method agree well with those computed by the interaction integral method. The maximum relative errors are 4.79, 3.75, and 4.3\% for the Mode I, Mode II, and Mode III SIFs, respectively. In general, the above relative errors are satisfactory and encouraging, and thus the validation and accuracy of the proposed method can be confirmed.

Then the effect of the quantity $a / W$ on the SIFs has been analyzed in detail based on the model of finite thermal barrier coating plate discussed above. It is of interest to find from Figure 9 that the location at which the maximum values of the SIFs occur is basically the surface point or the deepest point during the cooling shock process. It can be observed that as $a / W$ increases, the maximum values of the SIFs 


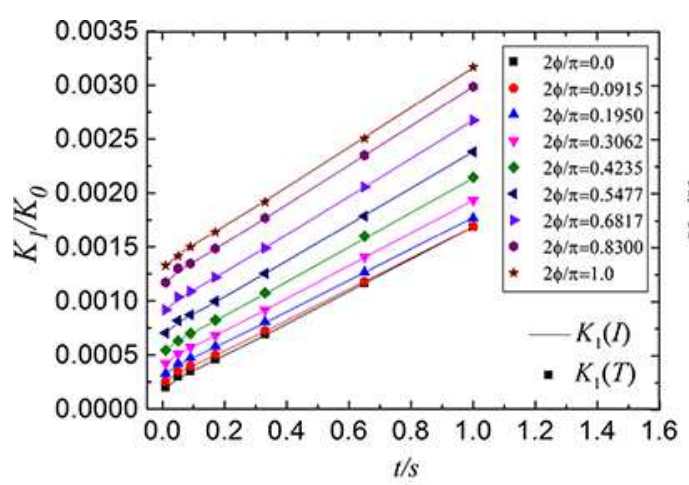

(a)

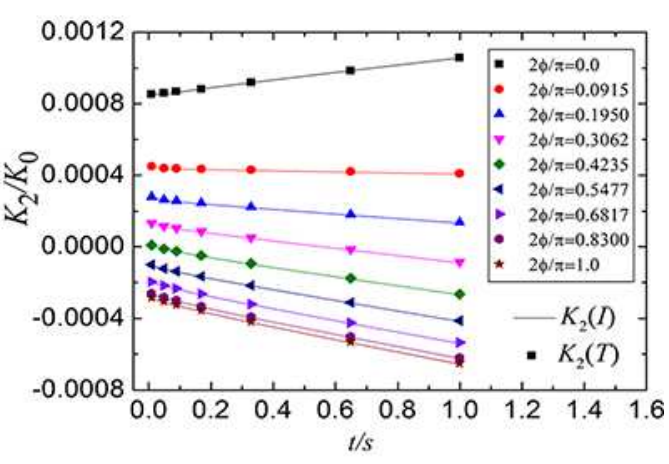

(b)

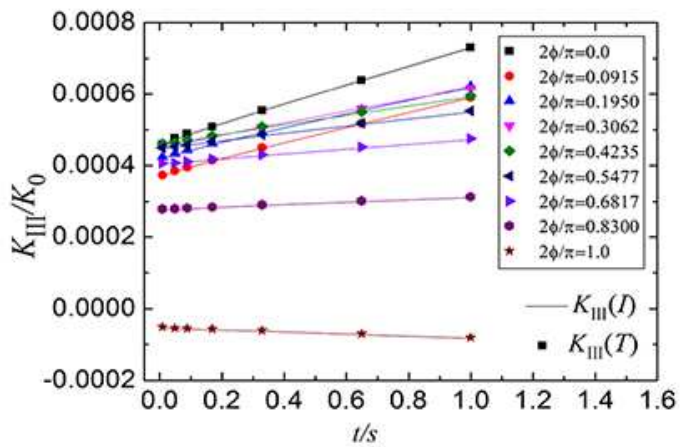

(c)

Figure 6. Comparison of stress intensity factors obtained from interaction integral and TWF methods for the case of instantaneous heat source. TWF: thermal weight function.

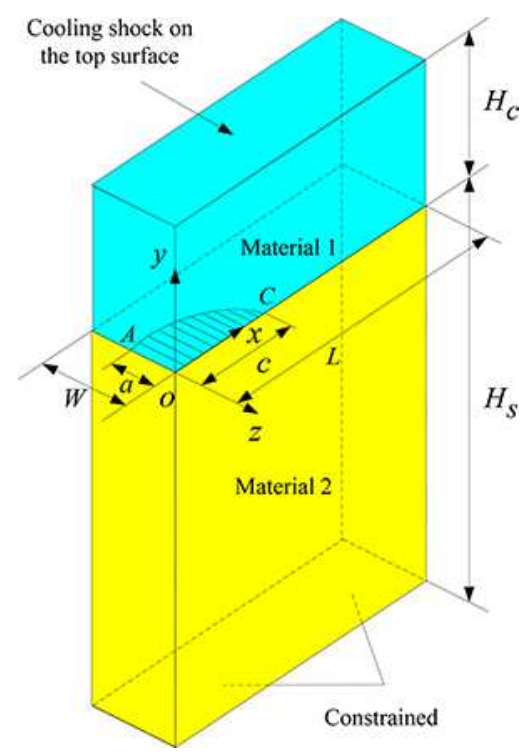

Figure 7. A bimaterial plate with a quarter-elliptical surface crack.

are also increased. That means the crack is more likely to propagate at the surface point or the deepest point. Meanwhile, the distributions of the normalized SIFs along the crack front vary with the increasing of $a / W$, which will alter the failure mode. The SIFs along 3D interface crack fronts vary complicated. 


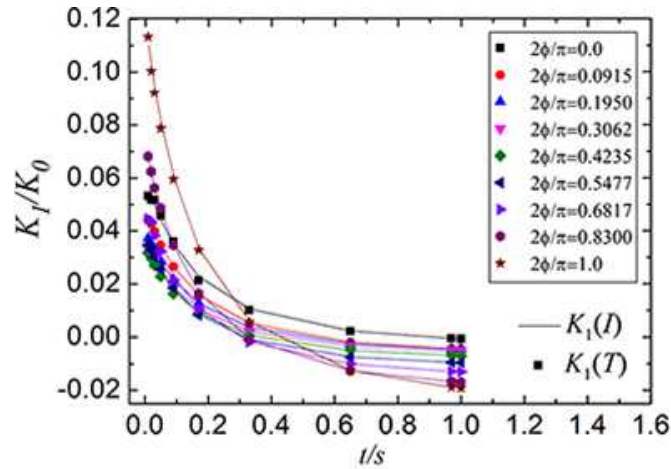

(a)

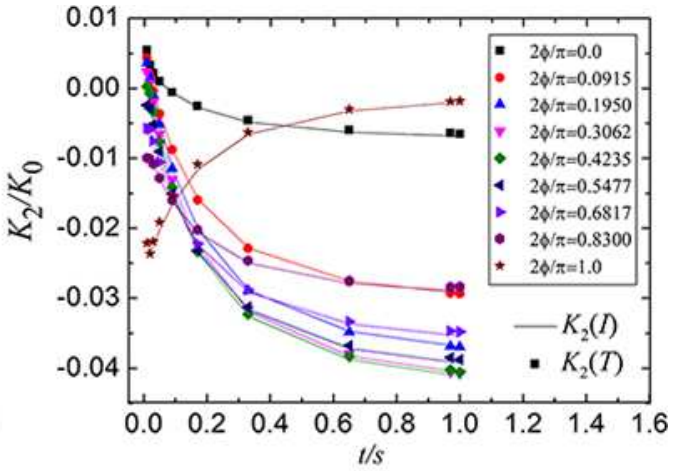

(b)

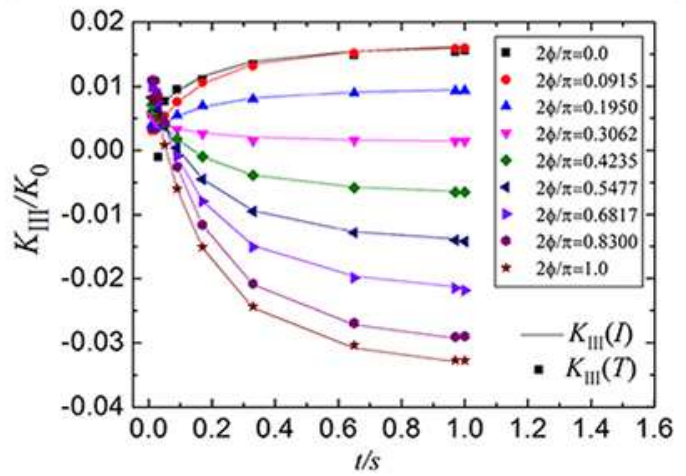

(c)

Figure 8. Comparison of stress intensity factors obtained from interaction integral and TWF methods for the case of thermal barrier coating system. TWF: thermal weight function.

Without analysis, it is almost impossible to predict the time and the location of the maximum SIFs, which requires to investigate the distributions of SIFs along the interface crack fronts in detail to accurately evaluate the safety of a 3D cracked body subjected to thermal load.

Actually, the proposed 3D TWF method is convenient and effective to deal with transient thermal problems, especially for lots of time instants. If such a problem is solved by the interaction integral method, a large amount of computation work will usually be needed. Most of the computation time will be spent on the repeated analyses of stress or displacement fields. However, little calculation amount will be involved if the 3D TWF method is employed. Taking the case of semi-infinite plate system presented above, for example, there are 100 time instants during the procedure of cooling shock, if the interaction integral method is applied to calculate all SIFs, 100 times of repeated stress computations must be performed, and the total time spent on stress analyses is about $2600 \times 100 \mathrm{~s}$ which is about $72 \mathrm{~h}$ on a microcomputer (Intel (R) Core (TM) i7 CPU 870 @ $2.93 \mathrm{GHz}$ ). However, only four times of computations will be needed using the 3D TWF method (thrice for displacement analyses for the reference loading system $(r)$, one time for TWF calculation), and the total time is about $2600 \times 3+450$ $\mathrm{s}$ which is about $2.3 \mathrm{~h}$. When compared with the interaction integral method, the $3 \mathrm{D}$ TWF method improves the computational efficiency by 31.3 times for achieving the same level of numerical accuracy. As can be seen, though the integration should be carried out over the whole volume of the cracked body and the whole boundary during the TWF calculation, by means of a series of integrated programs, the proposed TWF method is suitable for dealing with transient thermal problem. The high computational efficiency of the TWF method will be more obvious with increasing the number of elements, time instants, and shock loads. It should be noted that for steady state analysis, the advantage of high computational efficiency will vanish because the fundamental reference loading systems are needed to calculate thrice. 


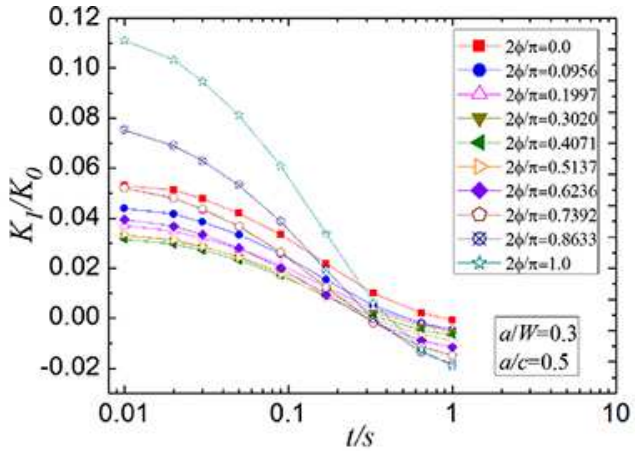

(a)

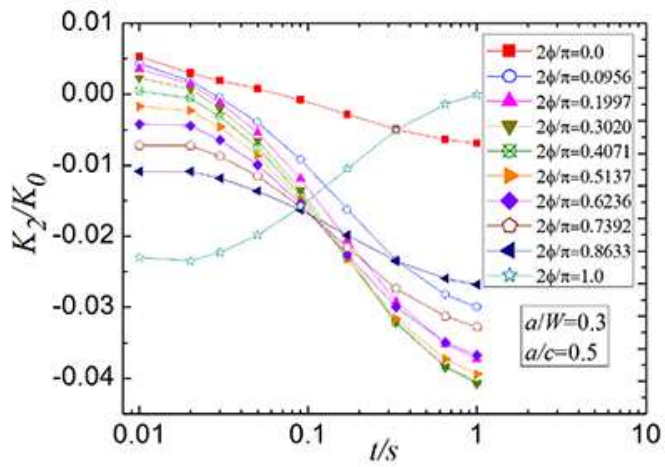

(c)

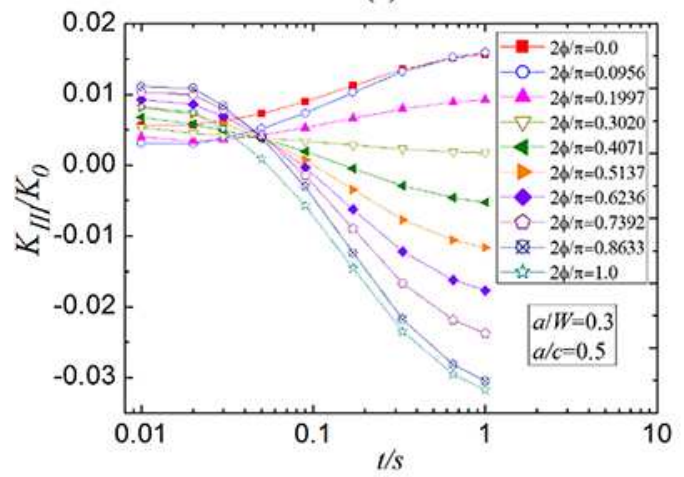

(e)

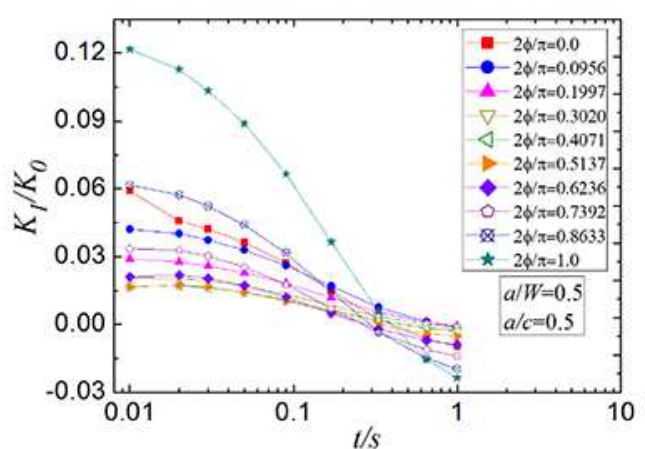

(b)

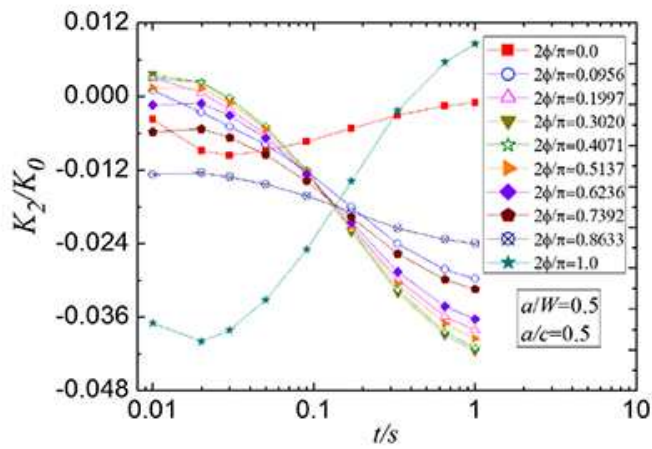

(d)

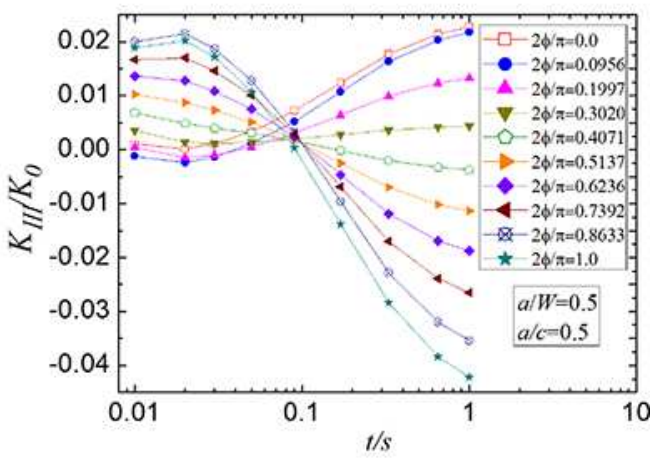

(f)

Figure 9. Variation of Mode I, Mode II, and Mode III stress intensity factors for different ratios of $a / W$ with respect to time.

\section{Conclusion}

In the present study, a 3D TWF method is proposed to analyze elliptical interface crack problems of bimaterial structures under transient thermal loadings. As the partial derivatives of the first invariants of the stress tensor with respect to the crack length are difficult to evaluate directly, the finite element implementation of the 3D TWF method is presented. As a part of finite element implementation of the 3D TWF method, the multiple virtual crack extension technique is applied to solve the basic equation. Several representative examples are investigated to demonstrate the accuracy of the 3D TWF method. In addition, the effect of the crack depth on the SIFs has been analyzed in detail using this method. 
The present investigation shows that

(1) The maximum values of the SIFs occur at the surface point or the deepest point during the whole cooling process, and they increase with crack depth.

(2) The failure mode may be altered due to the distribution change of transient SIFs along 3D interface crack fronts. Using 3D TWF method, expensive time consumed for the repeated determinations of the distributions of stress or displacement fields for individual time instants can be avoided.

(3) The proposed 3D TWF method is of good accuracy and of high computational efficiency in prediction of SIFs for an elliptical interface crack subjected to transient thermal loading, especially for large amount of time instants.

\section{Funding}

This work was supported by the National Natural Science Foundation of China under Grant Nos. 11372280, 51275471, and 11232013, the Zhejiang Provincial Natural Science Foundation of China under Grant No. LY15E050016, and the Zhejiang Provincial Public Welfare Technology Application Research Projects under Grant No. 2016C31041.

\section{References}

1. A. L. Florence and J. N. Goodier, Thermal Stress Due to Disturbances of Uniform Heat Flow by an Insulated Ovaloid Hole, Journal of Applied Mechanics-Transactions of the ASME, vol. 27, pp. 635-639, 1960.

2. G. C. Sih, On the Singular Character of Thermal Stress Near A Crack Tip, Journal of Applied Mechanics-Transactions of the ASME, vol. 29, pp. 587-589, 1962.

3. E. J. Brown and F. Erdogan, Thermal Stress in Bonded Materials Containing Cuts on the Interface, International Journal of Engineering Science, vol. 6, pp. 517-529, 1968.

4. M. K. Kassir and G. C. Sih, Thermal Stress in a Solid Weakened by an External Circular Crack, International Journal of Solids and Structures, vol. 5, pp. 351-367, 1968.

5. L. C. Guo, N. Noda, and L. Z. Wu, Thermal Fracture Model for a Functionally Graded Plate with a Crack Normal to the Surfaces and Arbitrary Thermomechanical Properties, Composites Science and Technology, vol. 68, pp. 1034-1041, 2008.

6. L. C. Guo and N. Noda, Modeling Method for A Crack Problem of Functionally Graded Materials with Arbitrary Properties-Piecewise-Exponential Model, International Journal of Solids and Structures, vol. 44, pp. 6768-6790, 2007.

7. L. C. Guo, Z. H. Wang, and N. Noda, A Fracture Mechanics Model for a Crack Problem of Functionally Graded Materials with Stochastic Mechanical Properties, Proceedings of the Royal Society A, vol. 468, pp. 2939-2961, 2012.

8. A. M. Bregman and M. K. Kassir, Thermal Fracture of Bonded Dissimilar Media Containing a Penny-Shaped Crack, International Journal of Fracture, vol. 10, pp. 87-98, 1974.

9. K. Y. Lee and C. W. Shul, Determination of Stress Intensity Factors for an Interface Crack under Vertical Uniform Heat Flow, Engineering Fracture Mechanics, vol. 40, pp. 1067-1074, 1991.

10. L. C. Guo and N. Noda, Investigation Methods for Thermal Shock Crack Problems of Functionally Graded MaterialsPart I: Analytical Method, Journal of Thermal Stresses, vol. 37, pp. 292-324, 2014.

11. N. Noda and L. C. Guo, Thermal Shock Analysis for a Functionally Graded Plate with a Surface Crack, Acta Mechanica, vol. 195, pp. 157-166, 2008.

12. L. C. Guo, L. Z. Wu, T. Zeng, and L. Ma, Mode I Crack Problem for a Functionally Graded Orthotropic Strip, European Journal of Mechanics-A/Solids, vol. 23, pp. 219-234, 2004.

13. A. B. Zhang and B. L. Wang, Contact Zone Approach for an Electrically Impermeable Crack in Piezoelectric Materials Under Thermal-Mechanical Loading, Journal of Thermal Stresses, vol. 35, pp. 749-765, 2012.

14. B. L. Wang and O. P. Niraula, Transient Thermal Fracture Analysis of Transversely Isotropic Magneto-Electro-Elastic Materials, Journal of Thermal Stresses, vol. 30, pp. 297-317, 2007.

15. T. Ikeda and C. T. Sun, Stress Intensity Factor Analysis for an Interface Crack between Dissimilar Isotropic Materials under Thermal Stress, International Journal of Fracture, vol. 111, pp. 229-249, 2001.

16. L. Banks-Sills and O. Dolev, The Conservative M-Integral for Thermal-Elastic Problems, International Journal of Fracture, vol. 125, pp. 149-170, 2004.

17. L. Banks-Sills and C. Ishbir, A Conservative Integral for Bimaterial Notches Subjected to Thermal Stresses, International Journal for Numerical Methods in Engineering, vol. 60, pp. 1075-1102, 2004.

18. D. B. P. Huynh and T. Belytschko, The Extended Finite Element Method for Fracture in Composite Materials, International Journal for Numerical Methods in Engineering, vol. 77, pp. 214-239, 2009.

19. M. Samimi, J. A. W. van Dommelen, and M. G. D. Geers, An Enriched Cohesive Zone Model for Delamination in Brittle Interfaces, International Journal for Numerical Methods in Engineering, vol. 80, pp. 609-630, 2009. 
20. S. E. Ashari and S. Mohammadi, Delamination Analysis of Composites by New Orthotropic Bimaterial Extended Finite Element Method, International Journal for Numerical Methods in Engineering, vol. 86, pp. 1507-1543, 2011.

21. H. J. Yu, L. Z. Wu, L. C. Guo, Q. L. He, and S. Y. Du, Interaction Integral Method for the Interfacial Fracture Problems of Two Nonhomogeneous Materials, Mechanics of Materials, vol. 42, pp. 435-450, 2010.

22. L. Z. Wu, H. J. Yu, L. C. Guo, Q. L. He, and S. Y. Du, Investigation of Stress Intensity Factors for an Interface Crack in Multi-Interface Materials Using an Interaction Integral Method, Journal of Applied Mechanics-Transactions of the ASME, vol. 78, pp. 061007, 2011.

23. C. P. Jiang, X. F. Wu, J. Li, F. Song, Y. F. Shao, X. H. Xu, and P. Yan, A Study of the Mechanism of Formation and Numerical Simulations of Crack Patterns in Ceramics Subjected to Thermal Shock, Acta Materialia, vol. 60, pp. 4540-4550, 2012.

24. F. N. Guo, L. C. Guo, K. Huang, X. M. Bai, S. Y. Zhong, and H. J. Yu. An Interaction Energy Integral Method for T-Stress Evaluation in Nonhomogeneous Materials under Thermal Loading, Mechanics of Materials, vol. 83, pp. 30-39, 2015.

25. L. C. Guo, F. N. Guo, H. J. Yu, and L. Zhang, An Interaction Energy Integral Method for Nonhomogeneous Materials with Interfaces under Thermal Loading, International Journal of Solids and Structures, vol. 49, pp. 355-365, 2012.

26. K. Y. Lee and W. C. Baik, Boundary Element Analysis of Thermal Stress Intensity Factors for Interface Griffith and Cusp Cracks, Engineering Fracture Mechanics, vol. 47, pp. 909-918, 1994.

27. Y. L. Chung, C.Y. Chang, and C. C. Chien, Boundary Element Analysis of Interface Cracks Subjected to Non-Uniform Thermal Loading, International Journal of Fracture, vol. 110, pp. 137-154, 2001.

28. Y. Ochiai, Two-Dimensional Unsteady Thermal Stress Analysis by Triple-Reciprocity Boundary Element Method, Journal of Thermal Stresses, vol. 24, pp. 233-253, 2001.

29. G. I. Giannopoulos and N. K. Anifantis, Interfacial Steady-State and Transient Thermal Fracture of Dissimilar Media using the Boundary Element Contact Analysis, International Journal for Numerical Methods in Engineering, vol. 62, pp. 1399-1420, 2005.

30. R. Khandelwal and J. M. Chandra Kishen, Thermal Weight Functions and Stress Intensity Factors for Bonded Dissimilar Media Using Body Analogy, Journal of Applied Mechanics-Transactions of the ASME, vol. 78, pp. 061019, 2011.

31. Y. Y. Zhang, L.C. Guo, and N. Noda, Investigation Methods for Thermal Shock Crack Problems of Functionally Graded Materials-Part II: Combined Analytical-Numerical Method, Journal of Thermal Stresses, vol. 37, pp. 325-339, 2014.

32. C. H. Tsai and C. C. Ma, Thermal Weight Function of Cracked Bodies Subjected to Thermal Loading, Engineering Fracture Mechanics, vol. 41, pp. 27-40, 1992.

33. H. F. Bueckner, A Novel Principle for the Computation of Stress Intensity Factors, Zeitschrift für Angewandte Mathematik und Mechanik, vol. 50, pp. 529-546, 1970.

34. J. R. Rice, Some Remarks on Elastic Crack-Tip Stress Field, International Journal of Solids and Structures, vol. 8, pp. 751-758, 1972.

35. T. L. Sham, A Unified Finite Element Method for Determining Weight Functions in Two and Three Dimensions, International Journal of Solids and Structures, vol. 23, pp. 1357-1372, 1987.

36. A. R. Shahani and S. M. Nabavi, Transient Thermal Stress Intensity Factors for an Internal Longitudinal Semi-Elliptical Crack in a Thick-Walled Cylinder, Engineering Fracture Mechanics, vol. 74, pp. 2585-2602, 2007.

37. J. R. Rice, Elastic Fracture Mechanics Concepts for Interfacial Cracks, Journal of Applied Mechanics-Transactions of the ASME, vol. 55, pp. 98-103, 1988.

38. C. F. Shih and R. J. Asaro, Elastic-Plastic Analysis of Cracks on Bimaterial Interfaces: Part I-Small Scale Yielding, Journal of Applied Mechanics-Transactions of the ASME, vol. 55, pp. 299-316, 1988.

39. M. Gosz, J. Dolbow, and B. Moran, Domain Integral Formulation for Stress Intensity Factor Computation along Curved Three-Dimensional Interface Cracks. International Journal of Solids and Structures, vol. 35, pp. 1763-1783, 1998. 